\title{
ANISOTROPIC CRYSTALLISATION IN POLYPROPYLENE INDUCED BY DEFORMATION OF A NUCLEATING AGENT NETWORK
}

\author{
Aurora Nogales ${ }^{1}$, Geoffrey. R. Mitchell ${ }^{1}$ and A. S. Vaughan ${ }^{2}$ \\ 'IJ Thomson Physical Laboratory, The University of Reading, Reading RG6 6AF, UK \\ ${ }^{2}$ Dept. Electronics and Computer Science, University of Southampton, Highfield SO17 1BJ, UK
}

\begin{abstract}
Memory effects on polypropylene systems with differents amounts of a nucleating agent (Dybenzylidene Sorbitol DBS) have been studied by Wide Angle X ray scattering methods. It has been observed that deformation applied in polypropylene melts, where even small amounts of DBS are included, is templated into the crystalline state. After crystallising a sheared polypropylene/DBS melt, an anysotropic texture is observed by $\mathrm{X}$ ray scattering, whereas the crystals produced from sheared pure PP melts are randomly distributed. This fact is directly addressed how the DBS is spread into the polypropylene melts. Different concentration and deformation conditions are explored allowing to conclude that, below a certain temperature, the DBS self-organize into a three dimensional network producing the gelation of the PP melt. When the deformation is applied in this gel state, it is templated into the crystals, whereas when it is applied at temperatures above this self organisation, there is not memory of the deformation process when the sample crystallise.
\end{abstract}




\section{INTRODUCTION}

During processing of polymeric systems, a great variety of deformation flows is applied to the polymer melt. These deformation fields induce certain levels of anisotropy in the polymer melt. The structure obtained after solidification of a deformed melt may differ from that obtained upon crystallisation in quiescent conditions. The differences may appear depending on several aspects.

On one hand, the 'memory' retained in the solidified polymer from the manufacturing process depends on the nature of the applied flow field (shear, elongational ...) and its intensity. Elongational flows are considered to be more effective in terms of extending the macromolecular chains. Fundamental studies aimed to understand flow induced crystallisation from solution were performed by Keller and coworkers $(1,2)$. The development of shish-kebab morphologies from elongated dilute solutions was observed. When dealing with polymers crystallised from elongated melts, it has been generally observed that shish-kebab textures are the predominant texture of their morphology $(3,4,5)$. As compare to elongational flow, shear fields are considered to be weaker. However, even these weaker flows have the effect of perturbing the chain configuration. The presence of chain entanglements enables the shear fields to produce some chain extension, which take place between these topological constraints (6). Recently, several works have been presented where the effect of shear deformation on the crystallisation is studied $(7,8,9,10,11)$. In all these investigations, an intense shear pulse (shorter compared to the time taken for crystallisation) was applied at samples held at the crystallisation temperature and the subsequent crystallisation was studied by means of in-situ techniques.

On the other hand, the memory that the solidified processed polymer retain from the deformations applied in the melt, may depend also upon the molecular characteristics of the polymer itself. The key factor in this effect would be the relaxation behaviour of the polymeric chains in the melt. Recent works point out the importance of the molecular weight distribution on the shear induced crystallisation (9), and moreover, the role of the long molecules in the behaviour under shear of the overall polymer $(11,12)$. But the molecular weight distribution is not the only factor affecting the relaxational behaviour of the polymer melt. The presence of additives, may affect in a considerable way, both, the rheological and the crystallisation behaviour of the polymer, and hence, they may have a big influence on the morphology of the polymer after deformation on 
the melt. In particular, the addition of nucleating agents to a polymeric matrix provokes extensive changes in terms of crystallisation behaviour and morphology of the polymer. Under quiescent conditions, the presence of small quantities of these compounds (even below $1 \%$ in weight) provides an excess of heterogeneous nuclei and hence increases the crystallisation temperature of the polymer, decreases the spherulite sizes and changes the optical and mechanical properties of the polymer (13).

In the present work, the influence of a nucleating agent in the crystallisation from sheared polypropylene melts is studied by means of wide and small angle $\mathrm{X}$ ray scattering. Dybenzylidene sorbitol (DBS) is a low molecular mass organic molecule that promotes heterogeneous nucleation in polypropylene at temperatures below the melting point of the polymer $(14,15,16)$. The aim of the present paper is to establish the consequences of the presence of this particular nucleating agent, on the developed microstructure after crystallising from a deformed melt. In the works on shear-induced crystallisation mentioned above $(7,8,9,10,11)$, the shear field is applied at the crystallisation temperature. In the present paper, however, the shear deformation is applied at temperatures above the calorimetric melting temperature of the polymer. Therefore, the crystallisation from deformed melts is studied rather than the shear induced crystallisation. The effect of different shear conditions on the nucleated polymer is discussed, and on the basis of the obtained results, a model for the behaviour of the nucleating agent in the polypropylene melt is presented.

\section{EXPERIMENTAL PART.}

\section{Materials and sample preparation.}

The polymer matrixes studied in this work are polypropylene/ethylene copolymers, with trade name NOVOLEN (BASF plc.). According to previous studies on these samples (17) the average molecular mass values obtained by Gel Permeation Cromatography $(\mathrm{GPC})$ are $\mathrm{M}_{\mathrm{w}}=2.2 \times 10^{5}$ and $\mathrm{M}_{\mathrm{n}}=5.9 \times 10^{4}$. Also according to this study, the ethylene co-monomer content is 3.5\%. The nucleating agent Dibenzylidene sorbitol (DBS) was obtained from Milliken under the trade name of Millad 3905. The chemical formula of this compound is presented in fig 1 . It was added to the polypropylene matrix by solution-precipitation method. The precipitated solution was then filtered and allowed to dry for several hours. Final removal of the solvent was done by vacuum drying at $70^{\circ} \mathrm{C}$. The compounded sample obtained in such a way was then 
melt presse at $190^{\circ} \mathrm{C}$ in the shape of discs of $1 \mathrm{~mm}$ thickness and $18 \mathrm{~mm}$ in diameter, suitable for use in the shear experiments. The samples are referred in this work as iPPx, where ' $\mathrm{x}$ ' indicates the percentage in weight of DBS in the sample.

\section{Techniques}

The rheometer to generate the shear flow field is a parallel plate system developed in the University of Reading and described elsewhere (18). It has been designed to allow scattering experiments during controlled shear deformations. Fig. 2 shows a schematic of the shear cell, and also a map of the velocity field in the sample (bottom left). Two X-ray sources were used. For the steady-state measurements, X-ray radiation generated in the laboratory was employed. It is generated by using a Philips sealed tube with a copper anode target, mounted on a Hiltonbrooks DG2 X-ray generator operating at $1.6 \mathrm{~kW}$ driving a $40 \mathrm{~mA}$ current. A Huber plane model 151 graphite crystal monochromator and pinhole collimation are employed to produce a beam $(\lambda=1.542 \AA)$ of $\sim 1 \mathrm{~mm}$ diameter. Time resolved measurements were performed on beamline 16.1 at the Synchrotron Radiation Source at Daresbury (Cheshire, UK), using a beam $(\lambda=1.488 \AA)$ of $\sim 0.4 \mathrm{~mm}$ diameter.

X-ray scattering data were collected using the Area X-ray Imaging System (AXIS) also developed at the University of Reading (19) and coupled with the rheometer. It uses an X-ray camera of $3 \times 4 \mathrm{~cm}$, based on an intensified CCD architecture provided with chip integration, designed and manufactured by Photonic Science. The video signal is passed to a frame grabber, where incoming frames are co-added to provide a more intense image with reduced statistical fluctuation. The frame-grabbing device employed is a DT2867 integrated image processor manufactured by Data Translation, which is mounted inside the control PC of the AXIS system. Integration times for the diffraction patterns were $4 \mathrm{~min}$ for the laboratory source and $10 \mathrm{~s}$ at the synchrotron source. The AXIS system is highly automated and the user can program complete sequences of events to provide X-ray scattering data synchronized with changes to the sample environment (19).

DSC experiments were carried out with a Perkin-Elmer DSC2 instrument at a heating rate of $10 \mathrm{C} / \mathrm{min}$. The temperature was calibrated by using indium and zinc standards. The samples were encapsulated in aluminium pans, and the typical sample weights used in these experiments were about $7 \mathrm{mg}$. 


\section{RESULTS}

\section{Characterisation of the samples with nucleating agent.}

\section{Differential scanning calorimetry}

Fig. 3 shows the cooling thermograms of samples after melting them at $\mathrm{T}_{\mathrm{m}}=190^{\circ} \mathrm{C}$ for 3 minutes. As one may see, the minimum in the crystallisation exotermic peak is located around $\mathrm{T}_{\mathrm{c}}=102^{\circ} \mathrm{C}$ for the iPP0. The incorporation of only $0.3 \%$ of the nucleating agent to the polymer produces a shift of the crystallisation peak towards higher temperatures $\left(\mathrm{T}_{\mathrm{c}}=114^{\circ} \mathrm{C}\right)$. The crystallisation temperature as a function of the weight concentration of DBS has been represented in the inset in fig. 3. The maximum shift is achieved for iPP0.5. For higher concentrations of DBS, there is a saturation of the shift in $T_{c}$ and the addition of more DBS is not further efficient in terms of increasing the crystallisation temperature. Eye inspection of the samples reveals that the high DBS content samples (iPP5 and iPP20) are not transparent. Mitra et al (20) studied the effect of adding different DBS amounts to poly(ethylene therepthtalate) (PET) from the point of view of the transparency of the polymer films. They found that there is an increase in the temperature of crystallisation from the melt when the DBS is incorporated. This increase is very strong for small concentrations of DBS and it stabilises for higher concentration. The transparency of the PET films started decreasing for DBS concentration higher than 1\% wt. The same authors studied the size of the DBS agglomerate in amorphous polymer system (21). For the two amorphous polymers (polycarbonate and polystyrene) they found that the size of the agglomerate increases linearly with the concentration of DBS, regardless of the polymer matrix. In terms of melting behaviour, the addition of DBS to the polypropylene matrix did not modify the melting temperature and the particular system under investigation here is completely molten for $\mathrm{T}>155^{\circ} \mathrm{C}$.

\section{Wide angle $X$ ray scattering}

Fig. 4 shows the wide-angle X-ray patterns obtained for polypropylene samples with different amount of nucleating agent. As one may see, in all samples, the scattered intensity exhibits, as a function of $\mathrm{q}(\mathrm{q}=(4 \pi / \lambda) \sin (\theta))$, Bragg peaks indexed 110 $\left(\mathrm{q}=0.99 \AA^{-1}\right), 040\left(\mathrm{q}=1.21 \AA^{-1}\right), 130\left(\mathrm{q}=1.31 \AA^{-1}\right)$ and the overlapping $-131,111$ (approx. at $\left.\mathrm{q}=1.58 \AA^{-1}\right)$. These peaks indicate that the main crystalline structure of the sample is the $\alpha$ 
form of iPP (22) that is therefore not modified by the presence of the nucleating agent. However, the presence of the nucleating agent introduces a variation on the WAXS diffraction pattern of the polymer. For all the samples with DBS, regardless of the concentration, a small peak appears around $q=1.45 \AA^{-1}$. A recent work by Foresta et al. (23) has shown that the presence of nucleating agents in similar polypropylene systems enhances the apparition of $\gamma$ phase. The (117) reflection of the $\gamma$ phase of PP is located at $\mathrm{q}=1.42 \AA^{-1}$. Therefore, this extra peak may indicate a certain presence of $\gamma$ phase.

\section{Isothermal crystallisation of sheared nucleated polypropylene melts.}

The isothermal crystallisation processes from sheared polypropylene melts with and without DBS were followed in real time by wide angle X-ray scattering (WAXS). The same shearing-temperature program was applied to the samples and it is presented in fig. 2 , bottom right, by a continuous line. The sample was held at the melting temperature $\left(\mathrm{T}_{\mathrm{s}}=170^{\circ} \mathrm{C}\right)$ for 5 minutes in order to erase any memory on the preparation process. After this time, the shear field was started. When the shear was released, the sample was quenched at the crystallisation temperature $\left(T_{c}\right)$ and it was held at this temperature for a given time. In situ WAXS patterns of the crystallisation process were monitored. Fig. 5 shows a sequence of WAXS patterns obtained at different times during the isothermal crystallisation process for both iPP0 and iPP0.5 samples. As one may see from these sequences, after shearing the melt under similar conditions $\left(\mathrm{T}_{\mathrm{s}}=170^{\circ} \mathrm{C}, \dot{\gamma}=1 \mathrm{~s}^{-1}\right.$, $\gamma=120$ s.u.), the two samples behave differently. First, there are differences in the kinetic of crystallisation. In the iPP0.5 sample, after 4.5 minutes at the crystallisation temperature, it is possible to observe the onset of crystallisation, as revealed by the development of crystalline rings. For the iPP0 sample, after 9 minutes at the crystallisation temperature, the presence of crystalline reflections cannot be detected. To emphasise the different crystallisation behaviours, the integrated intensity of the 110 ring has been represented as a function of crystallisation time for both the iPP0 and IPP0.5 samples (Fig. 6). The integrated intensity is presented in arbitrary units. However, it is plotted in the same scale for iPP0 and iPP0.5 specimens. For the iPP0.5 sample (fig. 6 top), as expected for an isothermal crystallisation process $(24,25)$, after a certain induction time, the 110 -intensity $\left(\right.$ at $\mathrm{T}_{\mathrm{c}}=125^{\circ} \mathrm{C}$ ) exhibits a rapid increase followed by a much slower growth at longer times. Further decrease of the temperature (plotted in fig. 6 as filled dots) produces another increase of the 110 intensity that finally reaches a levelling off for temperatures close to room temperature. The situation is very different 
for iPP0 sample. It is worth remarking that the sample was sheared under exactly the same conditions as the iPP0.5 specimen. The integrated intensity as a function of crystallisation time at $\mathrm{T}=125^{\circ} \mathrm{C}$ does not exhibit any increase. Even after approx. 13 minutes. After this time, the sample was cooled down to room temperature and X-ray patterns were collected. The appearance of crystalline reflections occurred at approx. $100^{\circ} \mathrm{C}$. At this temperature, the 110 integrated intensity exhibits a sudden increase and it reaches a levelling off at lower temperatures. These results agree with the cooling scans obtained by DSC (fig. 3.a)

However, not only the crystallisation kinetics is different for iPP0 and iPP0.5 samples. The initial X-ray scattering patterns for the two samples show diffuse rings corresponding to the amorphous halo of the molten specimen. But, as crystallisation proceeds, in the case of the iPP0.5 sample, discrete reflections appear, corresponding, as mentioned above, to the Bragg reflections of the $\alpha$ form of iPP. The azimuthal intensity distribution of this Bragg rings, in the case of iPP0.5 specimen is clearly not homogeneous. As crystallisation time increases, these rings become more intense, and also more homogeneous.

However, the iPP0 sample, when it crystallise at lower temperatures, exhibit completely isotropic reflections, indicating that the crystals grown are randomly oriented. That means that, iPP0.5 sample displays a memory effect of the deformation applied on the melt, but iPP0 specimen does not.

\section{Effect of the amount of DBS in the iPP anisotropic structure.}

In order to establish the role of the amount of DBS on the anisotropy induced upon shearing, a series of experiments were carried out under the same shearing conditions ( $\mathrm{T}_{\mathrm{s}}=160 \mathrm{C}, \dot{\gamma}=20 \mathrm{~s}^{-1}, \gamma=120$ s.u. $)$ on the different iPP samples. The specimens were subsequently quenched. The WAXS patterns obtained after quenching the sheared melts, are presented in fig. 7. The azimuthal orientation distribution of the PP WAXS reflections indicates a certain degree of orientation in the polymer matrix, even for the samples with very low amount of DBS. The obtained anisotropic texture is the same in all cases, although different levels of orientation can be observed depending on the DBS concentration. In fig. 8 the intensities of the 110 and the 040 reflections have been presented as a function of the azimuthal angle for the different quenched samples. For all the specimens, regadless of the DBS concentration, the (110) intensity is more intense near the meridian (azimuthal angle $\alpha=0^{\circ}$ ) but also present some intensity concentrated in 
the equator $\left(\alpha=90^{\circ}\right)$. The (040) intensity exhibits a maximum in the equator $\left(\alpha=90^{\circ}\right)$ and a minimum in the meridian $\left(\alpha=0^{\circ}\right)$. But, although they all present the same texture, there are some differences. As one may see, there is a dependence of the 'amount' of orientation with the DBS concentration. With the exception of the highest amount of DBS (sample iPP20), the orientation increases with the concentration of the nucleating agent, as can be inferred from the stronger dependence of the intensity with the azimuthal angle. It is worth mentioning that, in this representation, the intensity for the sample iPP0 shows a straight line, for both reflections.

\section{Influence of the different shearing conditions on the obtained anisotropic structure.}

\section{Melt temperature}

A series of iPP0.5 samples were sheared at different melting temperatures, under the same shearing conditions $\left(\dot{\gamma}=20 \mathrm{~s}^{-1}, \gamma=120\right.$ s.u.). After the shearing deformation was finished, the samples were quenched to room temperature. The azimuthal variation of the (110) and the (040) reflections It can be observed that, samples sheared at high temperature do not retain any memory on the deformation applied in the melt, and the crystallised sample is completely isotropic, as indicated by the lack of azimuthal dependence in the WAXS intensity. However, when the shearing is performed at $\mathrm{T}_{\mathrm{s}}=170^{\circ} \mathrm{C}$ or below, a highly anisotropic X-ray pattern it is obtained, and the crystallised sample exhibits an oriented texture. Therefore, this sample exhibit a transition around $\mathrm{T}=170^{\circ} \mathrm{C}$, that is related to the state of the DBS in the polymer melted matrix.

\section{Shear rate}

To identify which is the influence of the shear rate on the induced anisotropy, different shear rates were applied to a series of iPP0.5 samples, with the same shear strain $(\gamma=20$ s.u. $)$. The shearing deformation was performed at melt temperatures where, from the experiments presented in the previous paragraph, the iPP0.5 samples were known to retain some memory after crystallisation. The experiments presented here were performed at $\mathrm{T}_{\mathrm{s}}=160^{\circ} \mathrm{C}$. After finishing the shear program the samples were quenched. All the samples exhibited an anisotropic texture, regardless of the shear rate applied. The intensity of the 110 reflection and the 040 reflection has been presented as a function of 
the azimuthal angle in fig. 10. The reflection intensity is more dependent on the azimuthal angle for the highest shear rates.

Shear strain

Different strains were applied to iPP0.5 samples, sheared at the same melting temperature and at the same shear rate $\left(\mathrm{T}_{\mathrm{s}}=160^{\circ} \mathrm{C}, \dot{\gamma}=1 \mathrm{~s}^{-1}\right)$. The samples were then quenched to room temperature. The azimuthal variations of the 110 and the 040 reflections have been presented in fig. 11 for different shear strains. For very low shear strain $\left(\gamma=5 \mathrm{~s}^{-1}\right)$, no orientation is found and the WAXS pattern of the sheared sample consists of an isotropic distribution of intensity. However, for higher shear strains, the WAXS patterns exhibit anisotropic distribution of intensity. It is possible to see however, that, the orientation does not keep on increasing with increasing shear strain. For the highest shear strain applied, the intensity is anisotropy in the WAXS pattern is reduced.

\section{Dependence of the orientation with the different shearing conditions and sample composition.}

In order to have estimation on the anisotropy generated by the different shearing conditions and in the different samples, a measured of it was obtained by calculating the area under the azimuthal curves (in this case we have used the azimuthal variation of the 110 reflection). The obtained number is not an absolute measured of the orientation, but it allows us to characterise the trends in the variation of it.

A summary of the dependence of the orientation with the different shearing conditions and sample composition has been presented in fig. 12 .

\section{DISCUSSION}

The present X-ray experiments aim to characterise the structural differences in the morphology obtained after crystallising sheared polypropylene melts with and without DBS as a nucleating agent. The isothermal crystallisations of a DBS-modified iPP melt (iPP0.5) and a non-modified one (iPP0) followed by the WAXS experiments presented in fig. 5 reveal that the presence of DBS in the melt produced two main effects. The first effect is intrinsic of its nucleating agent nature. It accelerates the crystallisation by decreasing the induction time. This finding is in agreement with previous studies (17) where it has been pointed out that the DBS increases enormously the amount of nucleation sites and in PP preventing the appearance of a spherulitic texture and producing uniform lamella morphology when the crystallisation occurs below a critical 
temperature $\left(\mathrm{T}_{\mathrm{c}}<128^{\circ} \mathrm{C}\right.$ for the present PP system). But the most striking result presented here is the fact that, the sheared crystallisable PP melt retain memory on the deformation applied only when DBS is present (see fig. 5). As observed in fig. 5, the molten system does not present an evident anisotropy. However, it must exist some extent of anisotropy in the nucleated melt, and this anisotropy is templated afterwards by the process of polymer crystallisation, producing obvious levels of anisotropy. Previous works on polyethylene $(26,27)$ revealed that shearing the melt leads to small levels of global orientation, and by crystallising this sheared melts, the orientation parameters increase by an order of magnitude. In the cited case $(26,27)$ the memory from the shear deformation in the melts retained by the crystallised sample depend upon the molecular characteristics of the polymer, specifically, its molecular weight distribution. However, in the particular case treated here, the memory effect is dependent upon the presence of the nucleating agent. Two possibilities might be considered. On one hand, one may speculate that, the orientation-memory imposed by the presence of the nucleating agent may be due to a shift in the temperature range where the crystallisation of polypropylene occurs. At the early stages of crystallisation, crystallisation can be viewed as a physical gelation process $(28,29)$. As the crystallisation temperature is decreased, the time to reach the gel point decreases exponentially with the undercooling $\left(T_{m}-T_{c}\right)(29)$. The addition of the nucleating agent would produce an enhancement of this behaviour. Therefore, in the $\mathrm{iPP} / \mathrm{DBS}$ system, at low temperatures (below $\mathrm{T}=170^{\circ} \mathrm{C}$ ) the melt may contain some nuclei that act as physical crosslinks, that one may not find in the pure iPP system in this range of temperature. However, if this were the case, the memory effect would also be observed when shearing iPP melts nucleated with other substances. This seems not to be the case, in similar experiments performed in iPP nucleated with a Borealis technique (30).

Recent studies have shown that DBS induces thermoreverible gelation in several common solvents $(31,32,33,34,35)$. It is believed that DBS self-assembles through hydrogen bonding, even at relatively low concentrations, forming a three-dimensional percolation network in low molecular weight solvents. The critical gel concentration and the morphology of the DBS network have been found to be highly dependent on the polarity of the solvent (31). Also, DBS produced physical gelation when small concentrations are added to polymer solvents like polydimethylsiloxane, polypropylene glycol and polypropylene oxide (36,37). Shepard et al. have reported evolution of the complex viscosity as a function of temperature for PP samples modified with different 
concentrations of 1,3:2,4-di-p-methylbenzylidene sorbitol (MDBS) (38). They observe a pronounced increase in the intrinsic viscosity upon cooling the polymer melts, before it crystallises. This increases maybe attributed to the formation of a network MDBS nanofibrils. The temperature at which this increase in viscosity occurs has been found to be MDBS concentration dependent. Therefore, the above result support the hypothesis of DBS-modified polymer system undergoing a similar sol-gel transition below a certain temperature. Below this temperature $\left(170^{\circ} \mathrm{C}<\mathrm{T}_{\mathrm{s}}<180^{\circ} \mathrm{C}\right)$, DBS self-assemble into some structures (probably a fibril network) that become oriented with the shear process. This oriented structures, even in very low concentration, template the orientation during the iPP crystallisation process as indicated by the azimuthal orientation distribution of the PP reflections.

Regardless of the DBS concentration, shearing the DBS-modified polymer melt induces anisotropy when the sample crystallise (fig. 7). The oriented texture that appears upon cooling those sheared melts is the same. The iPP innermost reflection, (110), is more intense near the meridian with some intensity in the equator. The (040) reflection is most intense in the equator, and for the highly aligned samples, it presents virtually zero intensity in the meridian. This WAXS texture is compatible with a model of cross-hatched structure (39) of parents and daughter lamellae, where the secondary or daughter lamellae are able to nucleate and grow from previously formed primary lamella (parent). An idealized model of this orientation is presented in fig. 13. This model would predict the (040) intensity on the equator, since the b-axis of both, parents and daughter lamellae is perpendicular to the flow direction. Also, the model would predict the (110) intensity corresponding to the parent lamellae to be concentrated in the equator, since the c-axis of the parent lamella is parallel to the flow direction. The (110) intensity for the daughter lamella would lie nearly on the meridian, according to this model, since the c-axis of the daughter lamella is nearly perpendicular to the flow direction.

Assuming the above model for the orientation of the crystals in the DBSmodified PP crystallised samples, it is possible to speculate that the DBS would form fibrils that, during the shear process, are aligned in the direction of the shear flow. These fibrils have a high surface area (40), and assist polymer chains to nucleate and form the primary lamella, through an epitaxial interactions mechanism. The PP chains that form the parent lamella are parallel to the axis of the filaments. Other indications of epitaxial interactions between the DBS filaments and polyolefins chains have been reported by Thierry et al (41). In support of the presented hypothesis, close inspection of the WAXS 
pattern for the iPP5 sample, crystallised after shearing, reveals a series of equatorial reflections at q values lower than the ones of the iPP reflections. These reflections can be also observed in the iPP1 sample (42) and can be attributed to the DBS fibrils which are oriented parallel to the flow direction. Also, small angle $\mathrm{X}$ ray scattering results obtained during shear of the DBS modified melts corroborate this model, and they will be treated in a subsequent paper. The fact that only a small fraction of the material responds to the flow field and can template the anisotropy to the whole polymer system can be explained on the basis of the above mentioned high surface area of the assemblies of DBS.

Within this model, it is possible to explain the behaviour of the oriented intensity obtained under different shearing conditions. First of all, the dependence of the orientation with the shearing temperature $\left(T_{s}\right)$ (fig. 12.b) favours the occurrence of a solgel transition in the DBS modified PP melt. At the transition temperature, the DBS self assembles and form a percolation fibril network that absorbs the deformation, and template it into the polymer crystals. At a given temperature below the transition temperature, deforming the melts at different shear rates induces anisotropy. However, for very low shear rates the orientation is very low, suggesting some sort of relaxation of the alignment of the DBS fibrils. However, for shear rates above a threshold, the orientation is almost independent of the shear rate (fig. 12.c). The orientation exhibits a more complicated dependence with the shear strain. Very small shear strains do not produce anisotropy. Intermediate shear strains produce high levels of anisotropy. However, for very high shear strains the orientation is reduced, suggesting a breakage of the fibril network due to excess of strain (fig. 12.d). The orientation also presents some dependence with the DBS concentration (fig. 12.a). As can be observed, for DBS concentrations up to $5 \%$, for the same shearing conditions, the orientation increases with DBS concentration. However, the sample iPP20 exhibit very low orientation when deformed under the same shearing conditions. This effect can be explained by taking into account that, at these high levels of DBS, the fibrils may be forming bundles, which are more difficult to become align with the shear flow. From the rheological point of view, the formation of these bundles may imply an enhancement of the viscosity of the polymer melt. Therefore, higher strain is required to disentangle the fibril network. 


\section{CONCLUSIONS.}

The deformation applied to iPP samples modified by the addition of very small quantities of di-benzylidene sorbitol (DBS) produces an anisotropic texture when the sample is crystallised. The results presented here indicate that the DBS form a three dimensional network at temperatures below a given transition temperature. Below this transition temperature, the DBS modified polymer melts can be envisioned as a gel system. When this gel system is sheared the orientation induced in the melt is templated and multiplied when the polymer crystallised. The proposed model consists of a system of DBS fibrils, which become aligned in the flow direction. These fibrils, due to their high surface area nucleate primary PP lamella, by means of epitaxial interactions, where the iPP chains are positioned parallel to the fibril axis. There is also a population of daughter lamella that grow onto the surface of the parent one.

\section{AKNOWLEDGEMENTS}

A.N. thanks the support of this research by a Marie Curie Fellowship of the European Union programme 'Human Potential' under contract number HPMF-CT-2000-00657.

\section{REFERENCES}

(1)Pope, D. P.; Keller, A. Colloid Polym. Sci. 1978, 256, 751.

(2)Miles, M. J.; Keller, A. Polymer 1980, 21, 1295.

(3) Keller, A.; Kolnaar, H. W. H. Mater. Sci. Technol. 1997, 18, 189.

(4) Eder, G.; Janeschitz-Kriegl, H.; Liedauer, S. Prog. Polym. Sci., 1990, 15, 629.

(5) Jerschow, P.; Janeschitz-Kriegl, H. Int. Polym. Process. 1997, $12,72$.

(6) Keller A, Kolnaar HWH. In: Meijer HEH, editor. Processing of polymers, vol. 18. 1997. p. 189-268. (chap. 4).

(7) Kumaraswamy G., Issaian A.M., Kornfield J.A., Macromolecules 1999, 32, 7537.

(8) Kumaraswamy G., Verma R.K., Issaian A.M., Wang P., Kornfield J.A., Yeh F., Hsiao B.S., Olley R.H., Polymer 2000, 41, 8931.

(9) Somani R., Nogales A., Hsiao B.S., Srinivas S., Tsou A.H., Sics I., Ezquerra T.A., Balta Calleja F.J., Macromolecules 2000;33(25):9385. 
(10) Somani R.H., Hsiao B.S., Nogales A., Fruitwala H., Srinivas S., Tsou A.H., Macromolecules 2001, 34, 5902.

(11) Nogales A., Hsiao B.S., Somani R., Srinivas S., Tsou A.H., Balta Calleja F.J., Ezquerra T.A., Polymer 2001, 42, 5247.

(12) Seki M., Thurman D.W., Oberhauser J.P., Kornfield J.A., Macromolecules 2002, 35, 2583.

(13) Jansen J., In Gächter and Müller, editor, Plastic Additive Handbook, Chapter 18.

(14) Kim Y.C., Kim C.Y., Kim S.C., Polym. Eng. Sci. 1991, 31, 1009.

(15) Kim C.Y., Kim Y.C., Kim S.C., Polym. Eng. Sci. 1993, 33, 1445.

(16) Fujiyama M., Wakino T., J. Appl. Polym. Sci., 1991, 42, 2739.

(17) Zhao Y, Vaughan AS, Sutton SJ, Swingler SG. Polymer 2001;42:6587.

(18) Nogales A., Thornley S.A., Mitchell G.R., J. Macrom. Sci: Phys. (In press)

(19) Pople JA, Mitchell GR, Chai CK. Adv X-Ray Anal 1995; 38: 531.

(20) Mitra D, Misra A. J Appl Polym Sci 1988; 36:387-402

(21) Misra A, Mitra D. Polymer 1988; 29:1990-1994

(22) J Varga, in: J Karger Kocsis ed., Polypropylene: Structure, blends and composites, Chapman\&Hall, London, 56-115, (1995)

(23) T. Foresta, S. Piccarolo, G. Goldbeck-Wood, Polymer 42 (2001) 1167.

(24) Schultz, J.M. Polymer Materials Science, Prentice Hall: New York, 1974.

(25) Zachmann, H.G., Wutz, C. Crystallisation of Polymers; Dosiere M. Ed. Kluwer Academic Publishers,

(26) Pople J.A., Mitchell G.R., Chai C., Polymer 1996; 37, 4187.

(27) Pople J.A., Mitchell G.R., Sutton S.J., Vaughan A.S., Chai C., Polymer 1999;40:2769.

(28) Schwittay C., Mours M., Winter H.H., Faraday Discuss. 1995, 101, 93.

(29) Pogodina N.V., Winter H.H., Macromolecules 1998, 31, 8164.

(30) A. Nogales, G.R. Mitchell, unpublished results

(31) Yamasaki S., Tsutsumi H., Bull. Chem.Soc. Jpn 1995, 68, 123

(32) Yamasaki S., Ohashi Y., Tsutsumi H., Tsujii K., Bull. Chem.Soc. Jpn 1995, 68, 146

(33) Yamasaki S., Tsutsumi H., Bull. Chem.Soc. Jpn 1994, 67, 906

(34) Yamasaki S., Tsutsumi H., Bull. Chem.Soc. Jpn 1994, 67, 2053

(35) Yamasaki S., Tsutsumi H., Bull. Chem.Soc. Jpn 1996, 69, 561

(36) Mercurio DJ, Spontak RJ, J. of Phys. Chem. B 2001, 105, 2091.

(37) Nunez CM, Whitfield JK, Mercurio DJ, Ilzhoefer JR, Spontak RJ Macrom. Symp. 1996, 100, 275. 
(38) Shepard TA, Delsorbo CR, Louth RM, Walborn JL, Norman DA, Harvey NG, Spontak RJ J. of Polym. Sci: Part B: Polym. Phys. 1997, 35, 2617.

(39) Lotz B, Wittmann C, J. of Polym. Sci: Part B: Polym. Phys. 1986, 24, 1541.

(40) Thierry A, Fillon B, Straupe C, Lotz B Wittmann JC Prog. Colloid Polym. Sci.1992, $87,28$.

(41) Thierry A, Straupe C, Lotz B Wittmann JC Polym. Commun. 1990, 31, 299.

(42) Nogales A, Mitchell GR, Pukanszky B, Turcsanyi B ACS Polym. Prepr.2002, 43, 246.

\section{LEGENDS TO FIGURES}

Figure 1: Chemical formula of the nucleating agent Dibenzylidene sorbitol (Millad 3905). Figure 2: Schematics of the parallel plate rheometer modified to fulfill the requirements for usage in the $\mathrm{X}$ ray beam line. Bottom left, velocity map across the sample thickness. Bottom right, temperature and shearing program applied to the samples.

Figure 3: Differential scanning calorimetry scans taken at a cooling rate of $10^{\circ} \mathrm{C} / \mathrm{min}$ of the pure polypropylene and the samples with different porcentages in weight (indicated in by the lables on the right) of DBS. The inset shows the crystallisation tempetature (temperature of the minimum in the heat transfer) as a function of the percentage in weight of the nucleating agent DBS.

Figure 4: Wide angle $\mathrm{X}$ ray scattered intensity as a function of $\mathrm{q}$, for isotactic polypropylene samples with different amount of DBS at room temperature, as indicated by the labels on the right hand side. The labels on top of the pattern indicate the index of the Bragg reflections corresponding to the $\alpha$ modification of iPP.

Figure 5: Wide angle X-ray scattering patterns obtained during the crystallisation process of the (a) iPP0 and (b) iPP0.5 sample obtained during the crystallisation process at different crystallisation times.

Figure 6: Integrated intensity under the 110 ring as a function of crystallisation time for (a) iPP0.5 and (b) iPP0 sample. Same shearing conditions in both cases

Figure 7: Wide angle X-ray scattering patterns for samples with different amount of DBS. All samples were sheared at $\mathrm{T}_{\mathrm{s}}=160^{\circ} \mathrm{C}, \dot{\gamma}=20 \mathrm{~s}^{-1}$ and $\gamma=120$ s.u. Samples quenched to room temperature after shear.

Figure 8: Intensity of the 110 and the 040 reflection as a function of the azimuthal angle for iPP samples with different concentration of DBS as indicated by the labels on the 
curves. All the samples have been sheared on the melt at $\mathrm{T}_{\mathrm{s}}=160^{\circ} \mathrm{C}, \dot{\gamma}=20 \mathrm{~s}^{-1}$ and $\gamma=120$ s.u.

Figure 9: Intensity as a function of the azimuthal angle for the 110 ring (a) and 040 ring (b) obtained from samples iPP0.5 crystallised after shearing at different temperatures $\left(\dot{\gamma}=20 \mathrm{~s}^{-1}, \gamma=120 \mathrm{~s} . \mathrm{u}\right)$.

Figure 10: Intensity as a function of the azimuthal angle for the 110 ring (a) and 040 ring (b) obtained from crystallised iPP0.5 samples after being sheared at different shear rates, and same shear strain and shearing temperature. $\left(T_{s}=160^{\circ} \mathrm{C}, \gamma=20\right.$ s.u. $)$

Figure 11: Intensity as a function of the azimuthal angle for the 110 ring (a) and 040 ring (b) obtained from crystallised iPP0.5 samples after being sheared with different strains, $\left(\mathrm{T}_{\mathrm{s}}=160^{\circ} \mathrm{C}, \dot{\gamma}=1 \mathrm{~s}^{-1}\right)$

Figure 12: Dependence of the orientation (measured as the area under the azimuthal 110 curve) with (a) the DBS concentration, (b) shear temperature, (c) shear rate and (d) shear strain.

Figure 13: Model to explain the oriented texture obtained by the WAXS patterns. 
Page 17 of 29

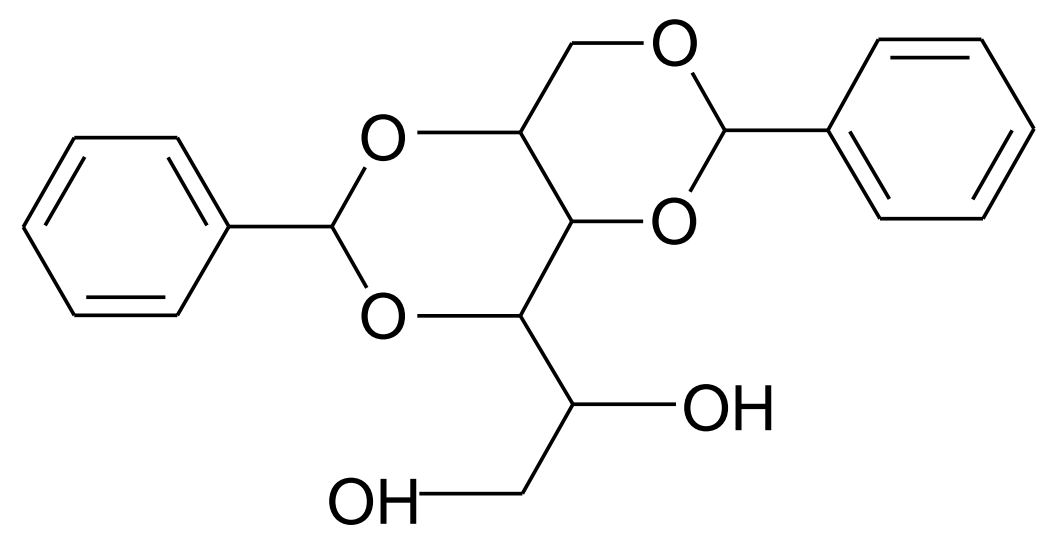

Fig.1 
Page 18 of 29
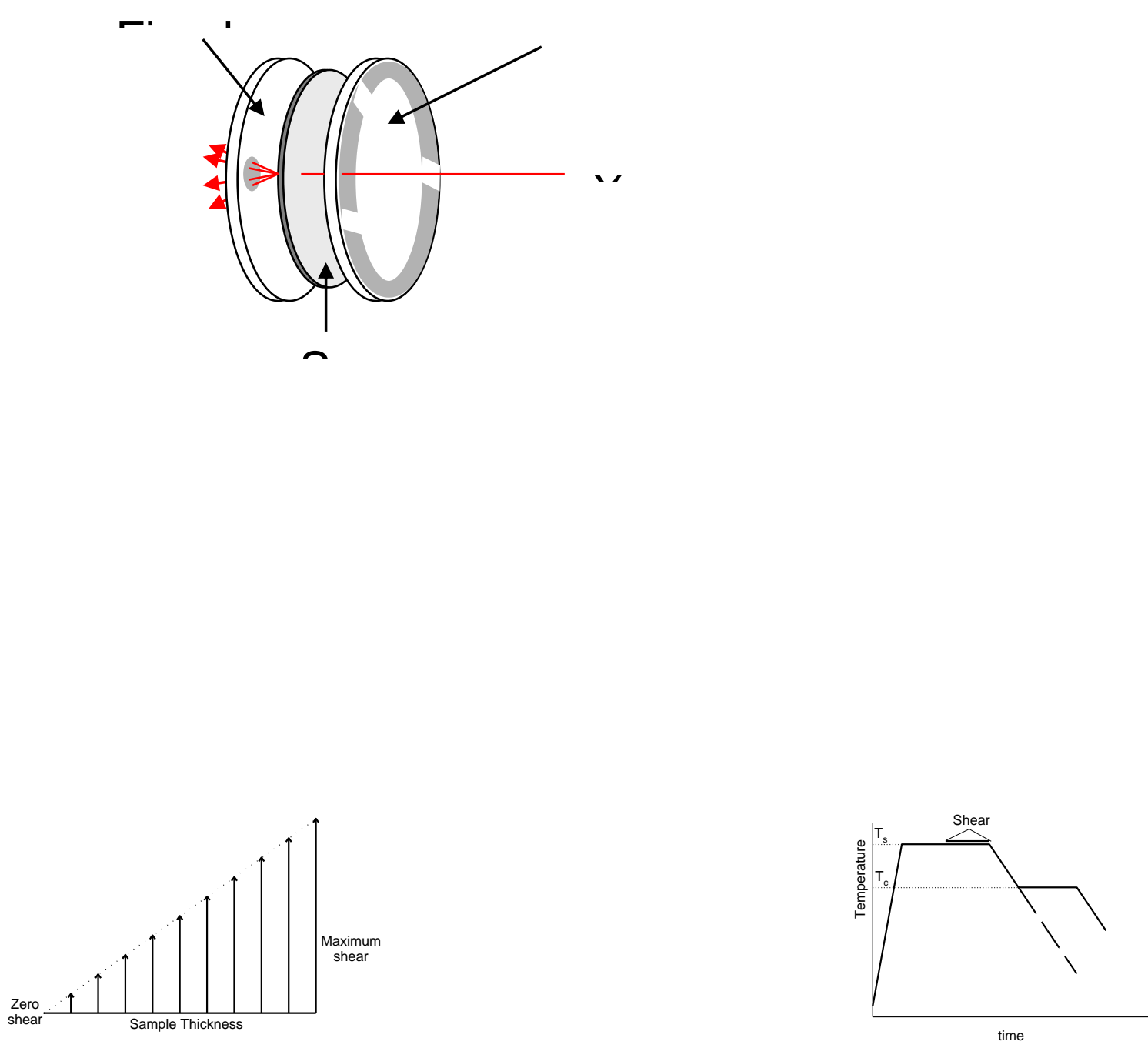

Fig.2 
Page 19 of 29

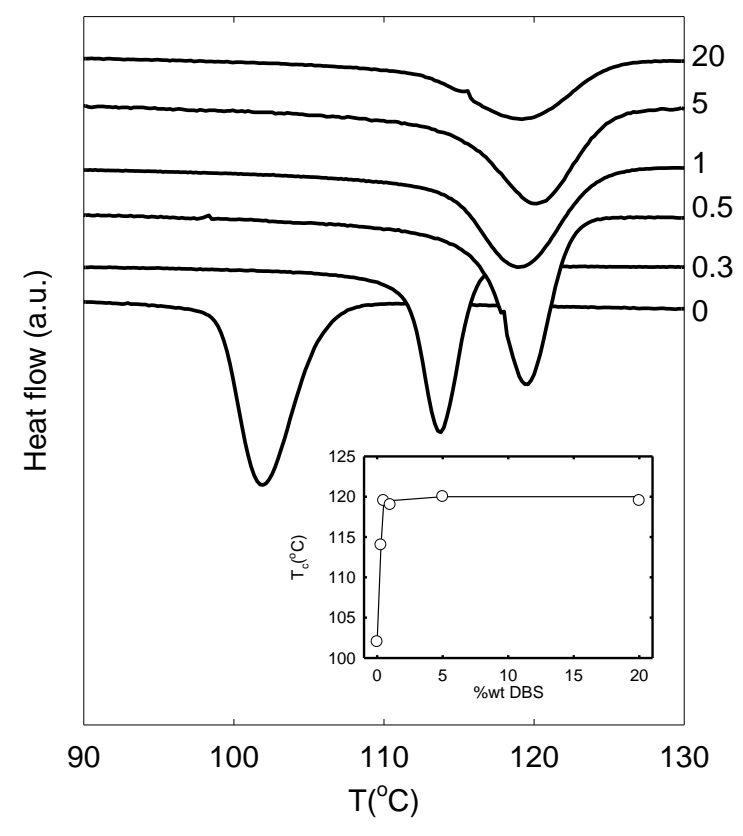

Fig.3 
Page 20 of 29

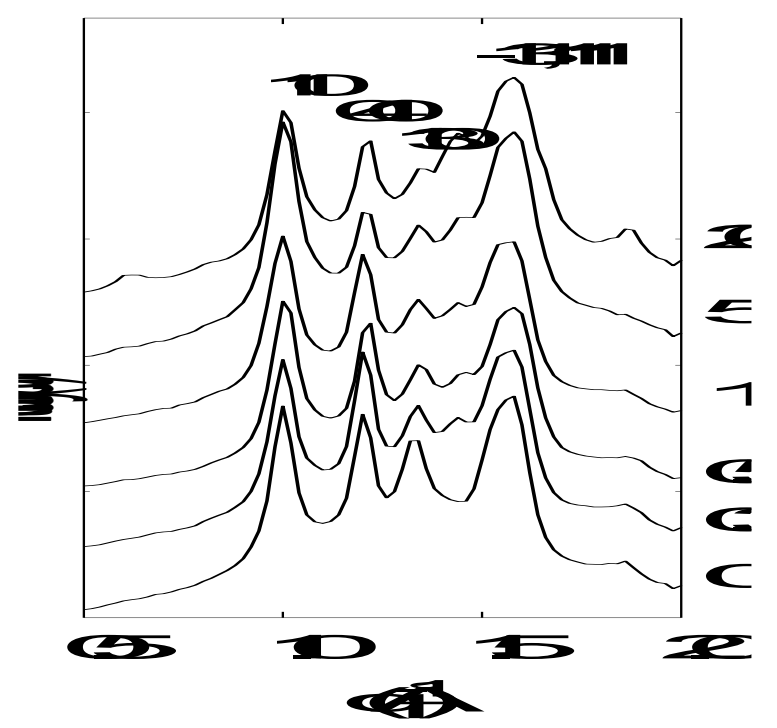

Fig.4 


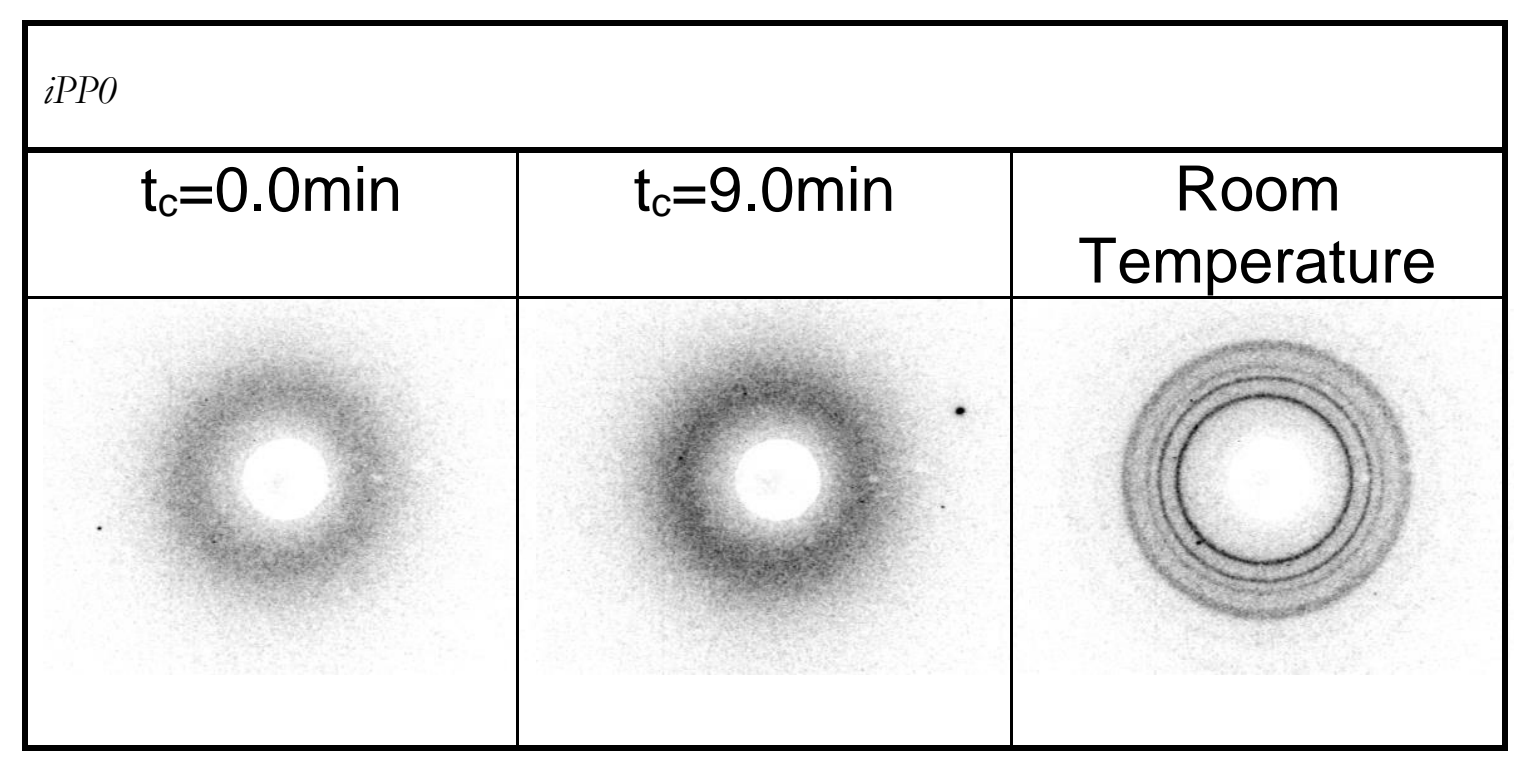

IPP0.5

\begin{tabular}{l|l|l}
$\mathrm{t}_{\mathrm{c}}=0.0 \mathrm{~min}$ & $\mathrm{t}_{\mathrm{c}}=4.5 \mathrm{~min}$ & $\mathrm{t}_{\mathrm{c}}=9.0 \mathrm{~min}$
\end{tabular}

Fig.5 

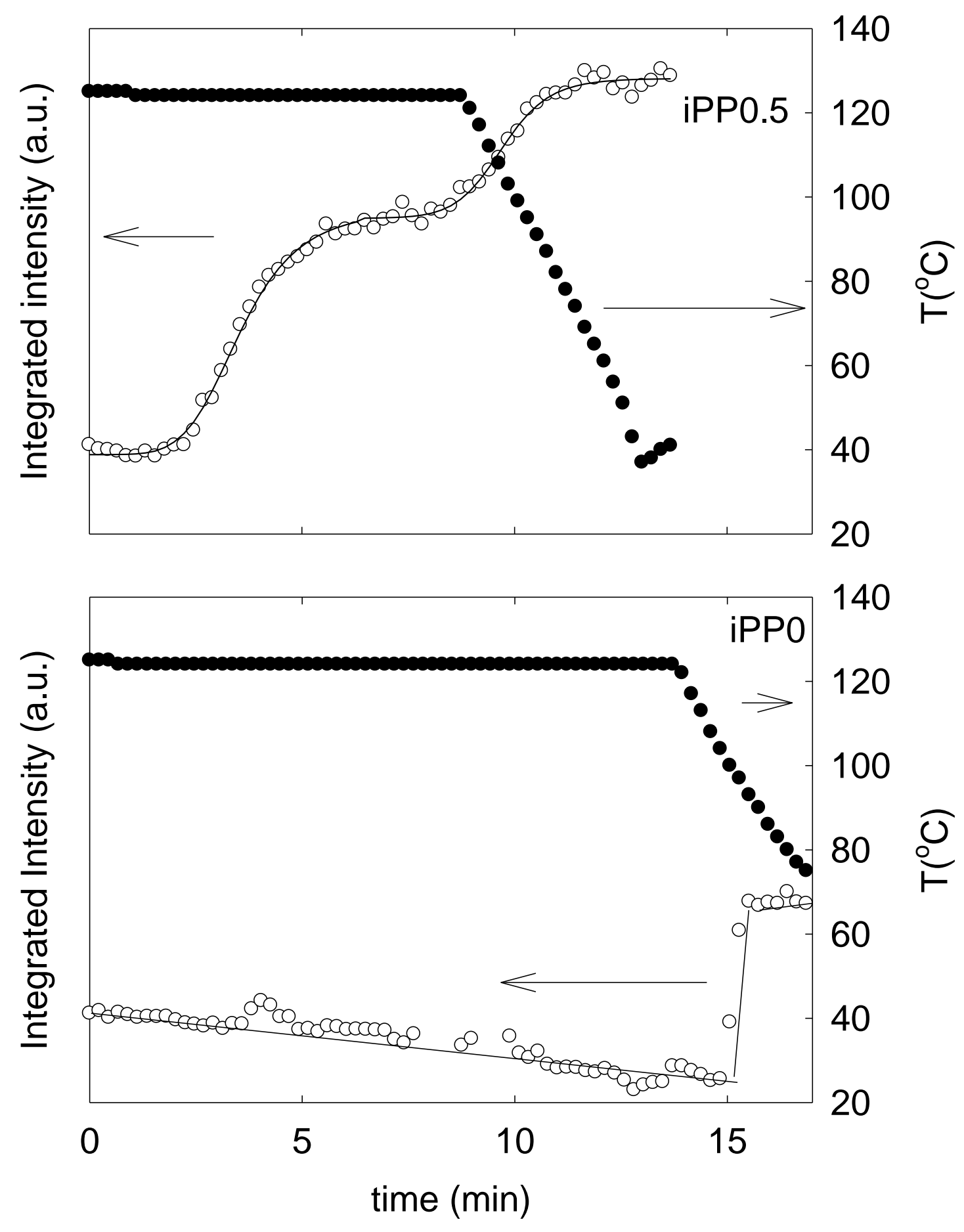

Fig. 6 
Page 23 of 29
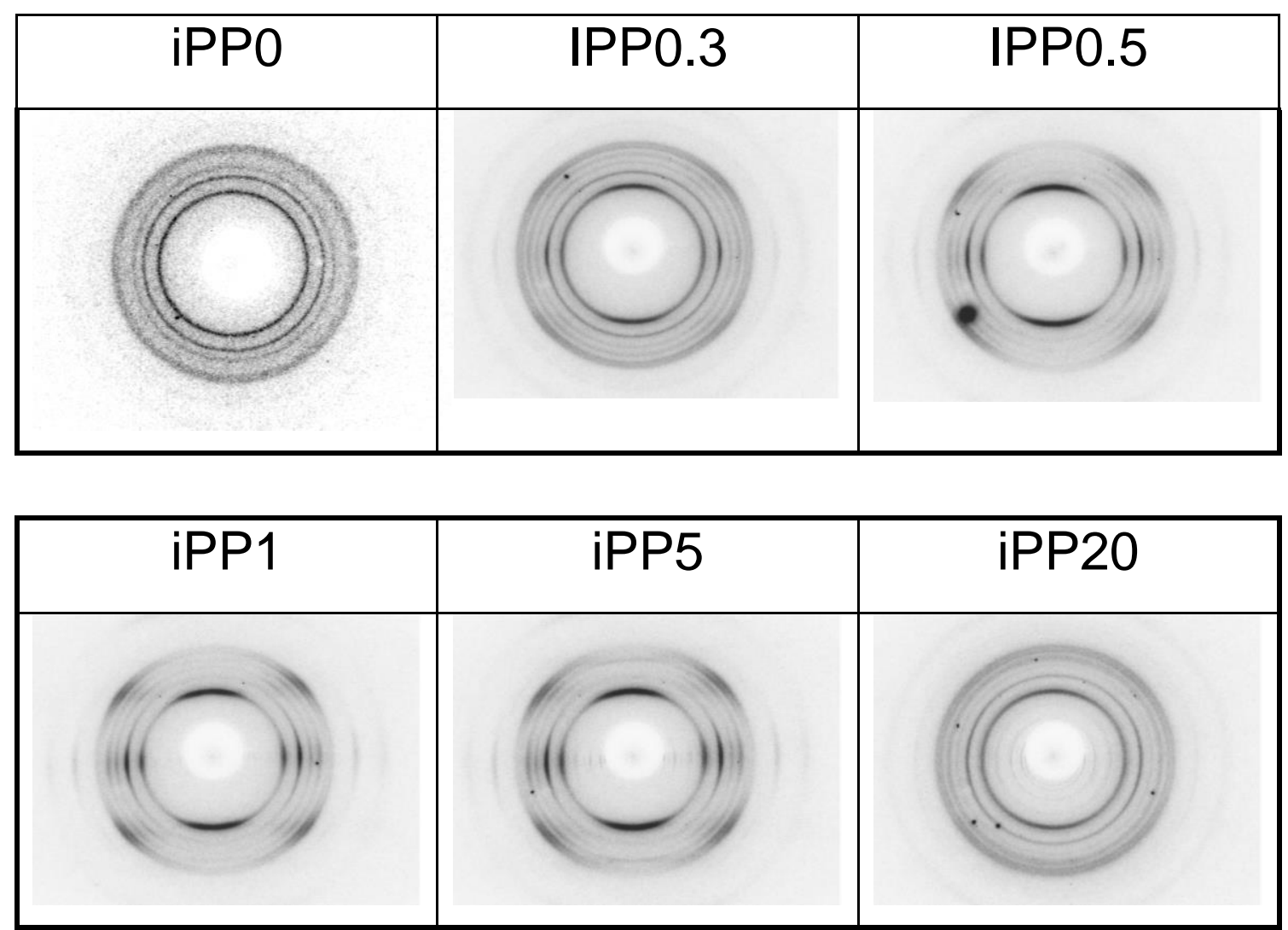

Fig.7 

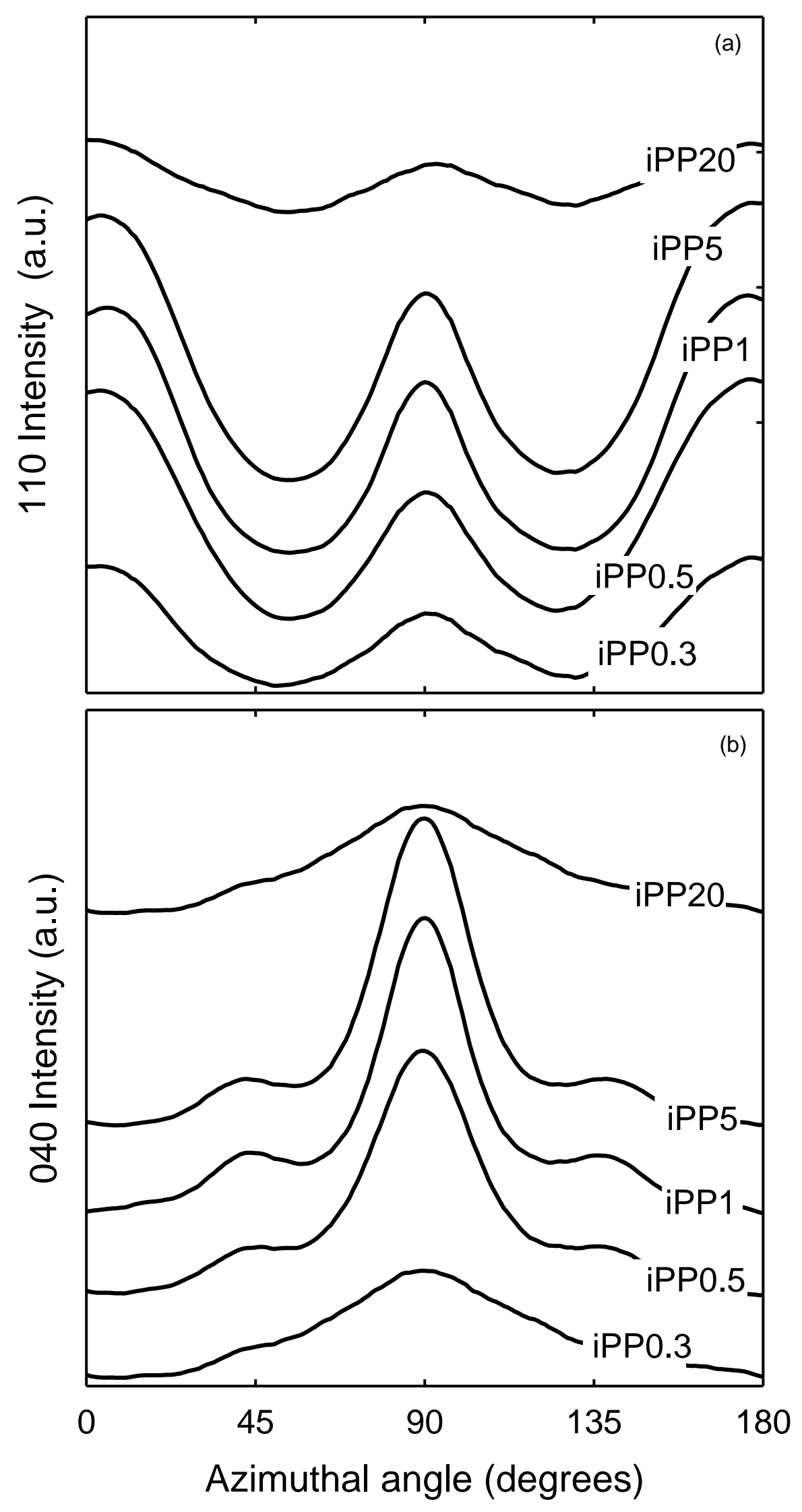

Fig.8 

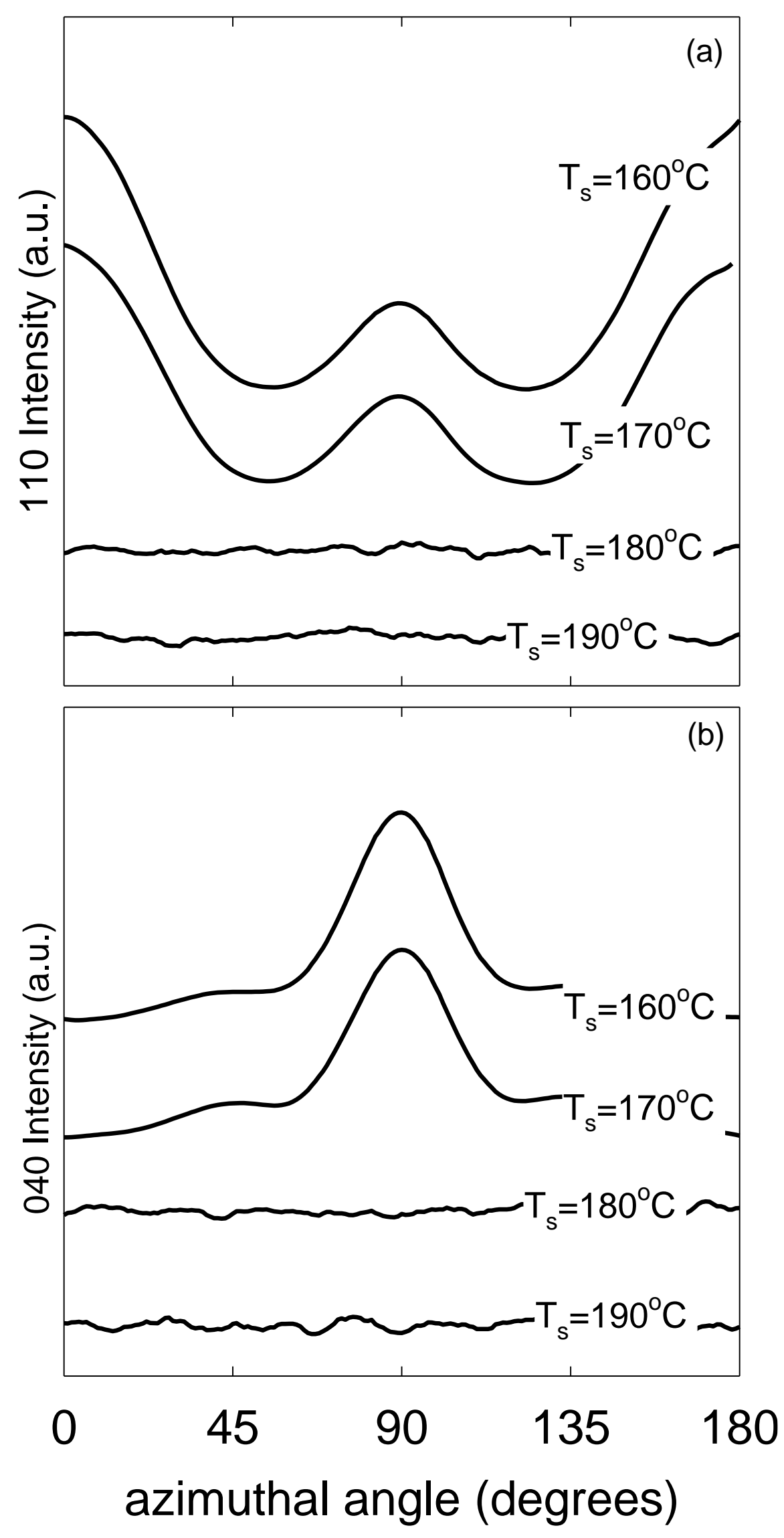

Fig.9 
Page 26 of 29

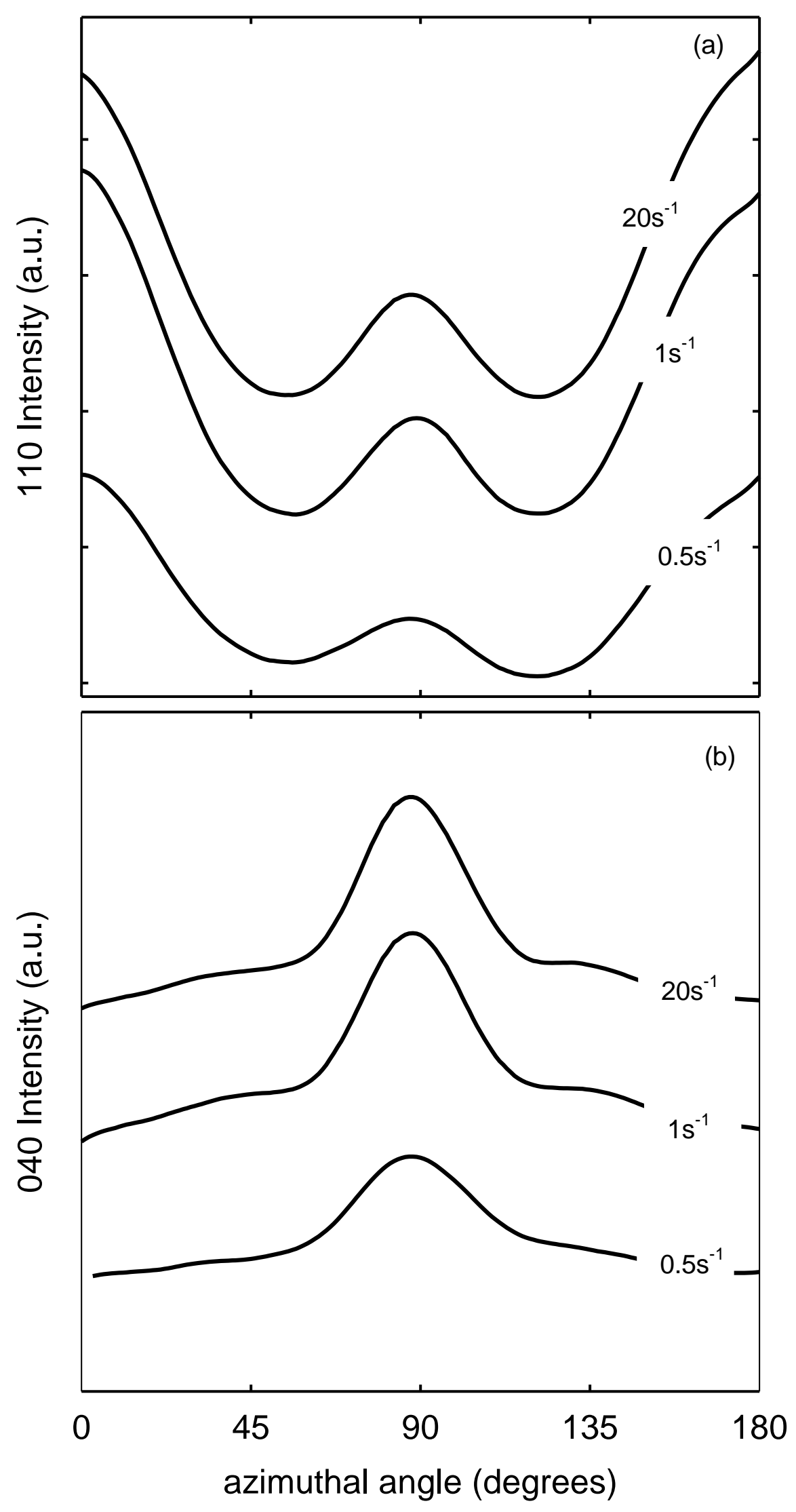

Fig.10 

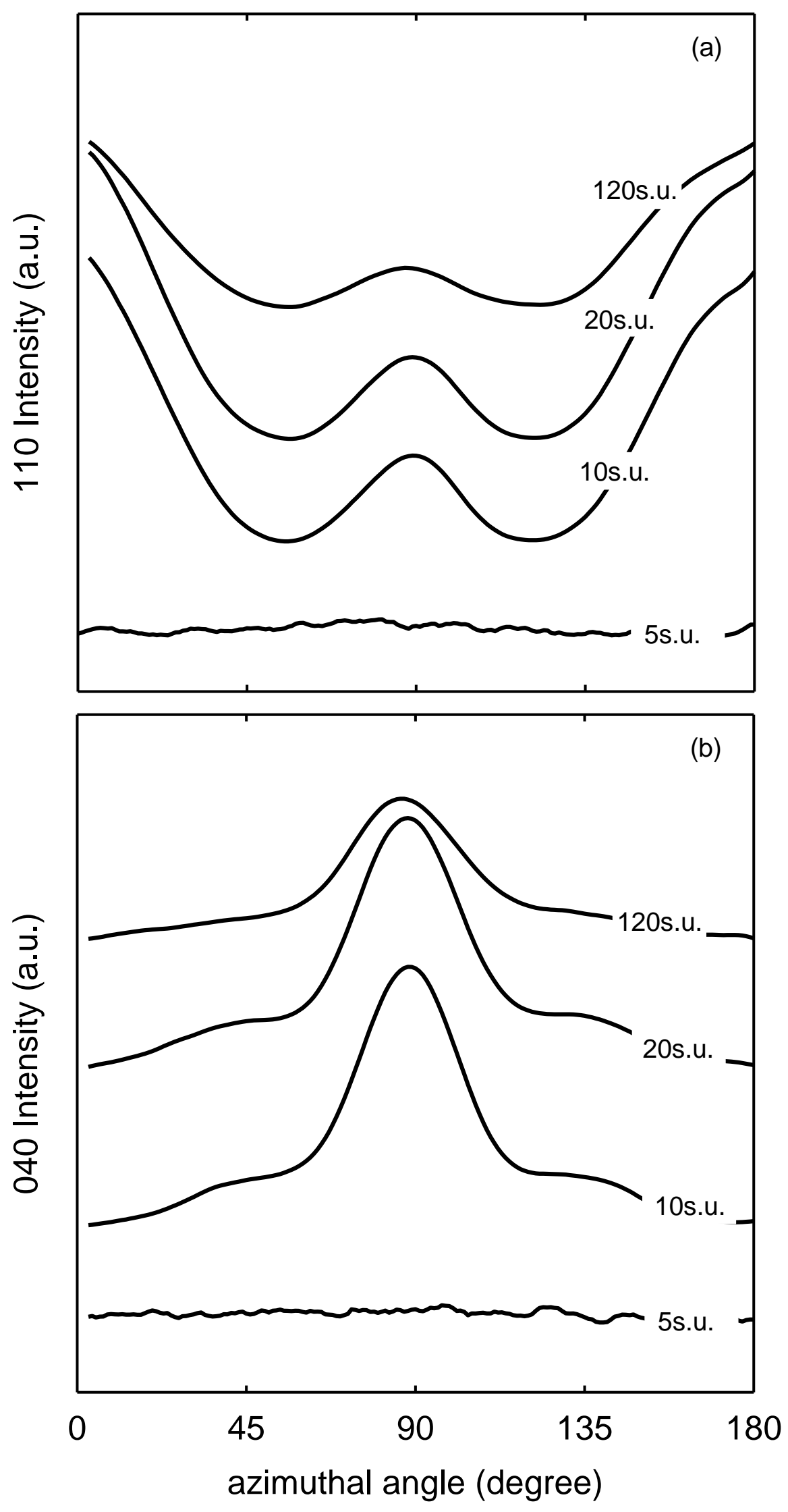

Fig.11 

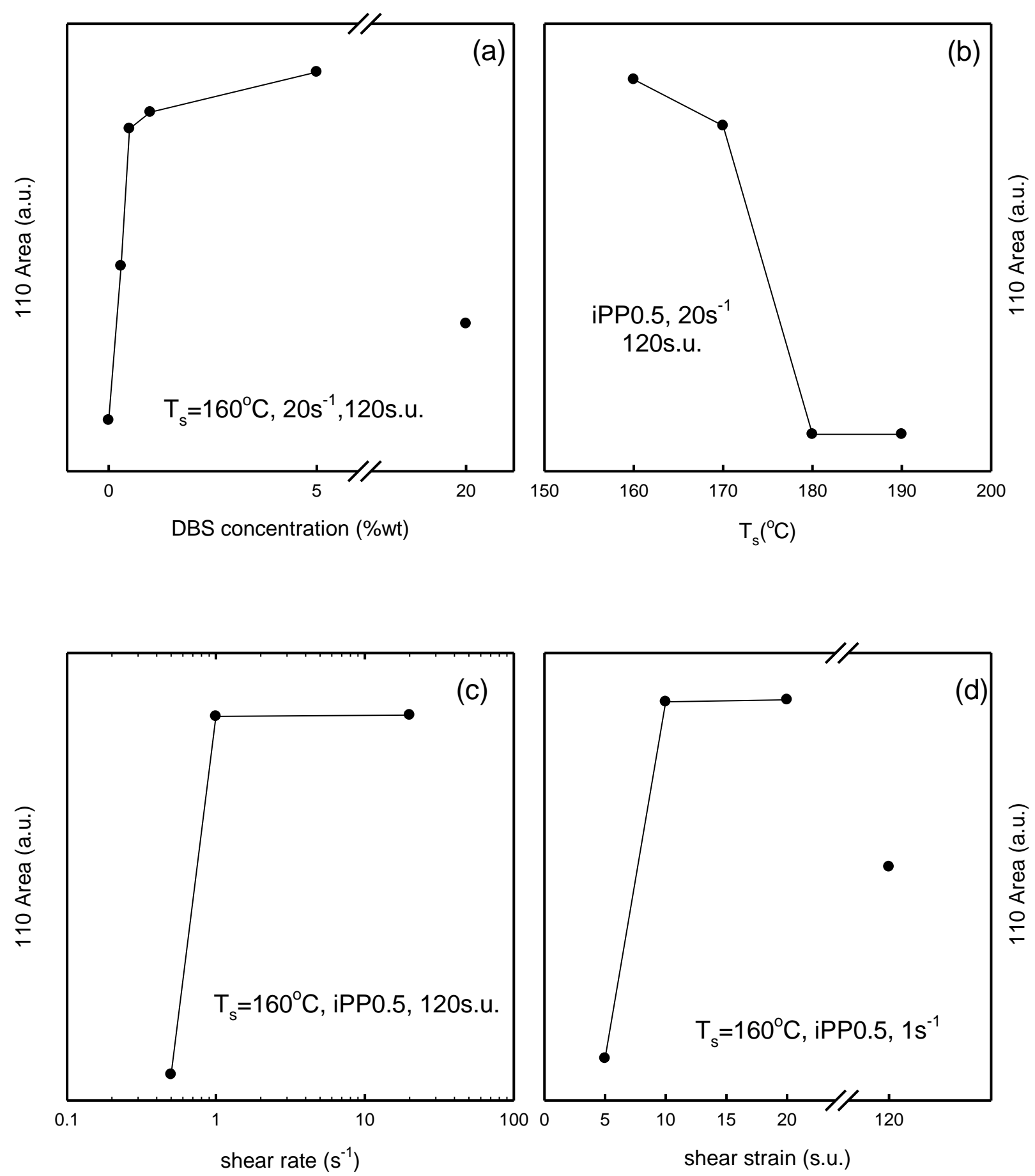

Fig.12 
Page 29 of 29

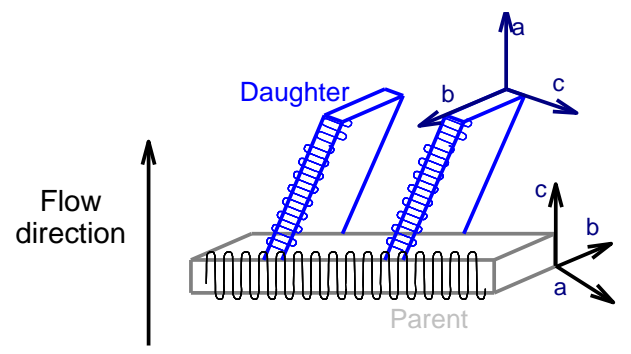

Fig.13 